\section{ADAPTABLE DENTAL UNIT}

Castellini's Duo dental unit provides the functional versatility necessary to a modern practice. Ergonomic and attractive, the Duo unit features the Dama Chair, which offers many benefits including preset and programmable movements and working positions.

It also benefits from increasing efficiency and accessibility, with two trays and multi-functional foot controls, intuitive lock function - if the Silent Power Turbine, or any instrument, is being used, then chair movement is locked for patient and dentist safety, and has active antibacterial upholstery for improved sanitation.

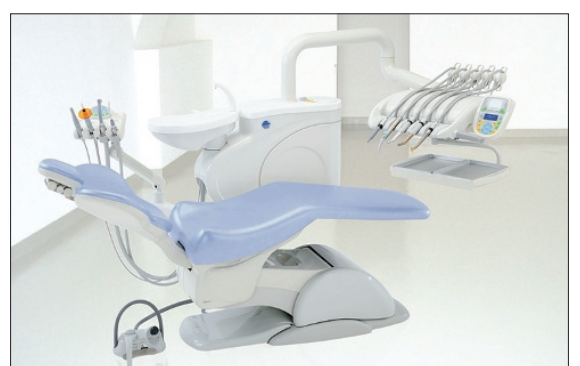

The unit also meets the modern practice's disinfection requirements with its easily detachable and autoclavable parts. It goes further by integrating a variable Auto-Sterilising mode that allows efficient cleaning cycles between patients or rigorous over-night disinfection.

Reader response number 59

\title{
SOLUTION FOR YOUR HYGIENE REQUIREMENTS
}

Infection control product manufacturer Schülke provide a wide range of products designed to offer solutions to your every hygiene requirement. These include Gigasept enzymatic, a unique three-enzyme cleaner and disinfectant. A safe and efficient product, gigasept enzymatic offers an alternative to manual scrubbing of instruments prior

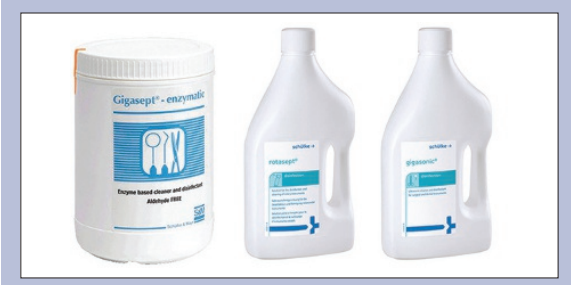

to autoclaving, and is effective against bacteria, fungi and viruses.

Rotasept, the ready-to-use solution for the cleaning and disinfection of rotary instruments, cleans burs, cutters, grinders, finishers and polishers. The product can be used in a static bath, or for quicker results inserted into an ultrasonic bath.

Finally, Gigasonic is a clear liquid cleaning product designed for use in ultrasonic baths. It ensures optimum appearance of instruments and prevents rusting and residue build up with just a five minute contact time.

Reader response number 60

\section{FULLY AUTOMATIC MIXING MACHINE}

MixStar-eMotion is DMG UK's latest generation fully automatic mixing machine, designed for use with their MixStar impression materials - Honigum-MixStar, Silagum-MixStar and StatusBlue.

The MixStar-eMotion possesses three different, electronically controlled speed settings that ensure consistent results even with more viscous materials. The top speed enables dispensing at twice the speed of its predecessor.

Setting and working time in the mouth can be adjusted with two timers and saved in programmes. The mixing process can be started with a simple press of a button or by using an optional footswitch, which can be plugged in for hands-free operation. With the aid of a serial interface, software can be updated anytime via the internet.

The unit is a fully automatic mixing machine designed to deliver perfectly mixed, homogenous, bubble-free impression material directly into the impression tray or syringe.

User-friendly, it is easy to operate at the touch of a button, enabling both of the operator's hands to remain free to hold the tray or syringe during filling. It offers quick and easy cartridge and mixing tip exchange when required.

Reader response number 61 